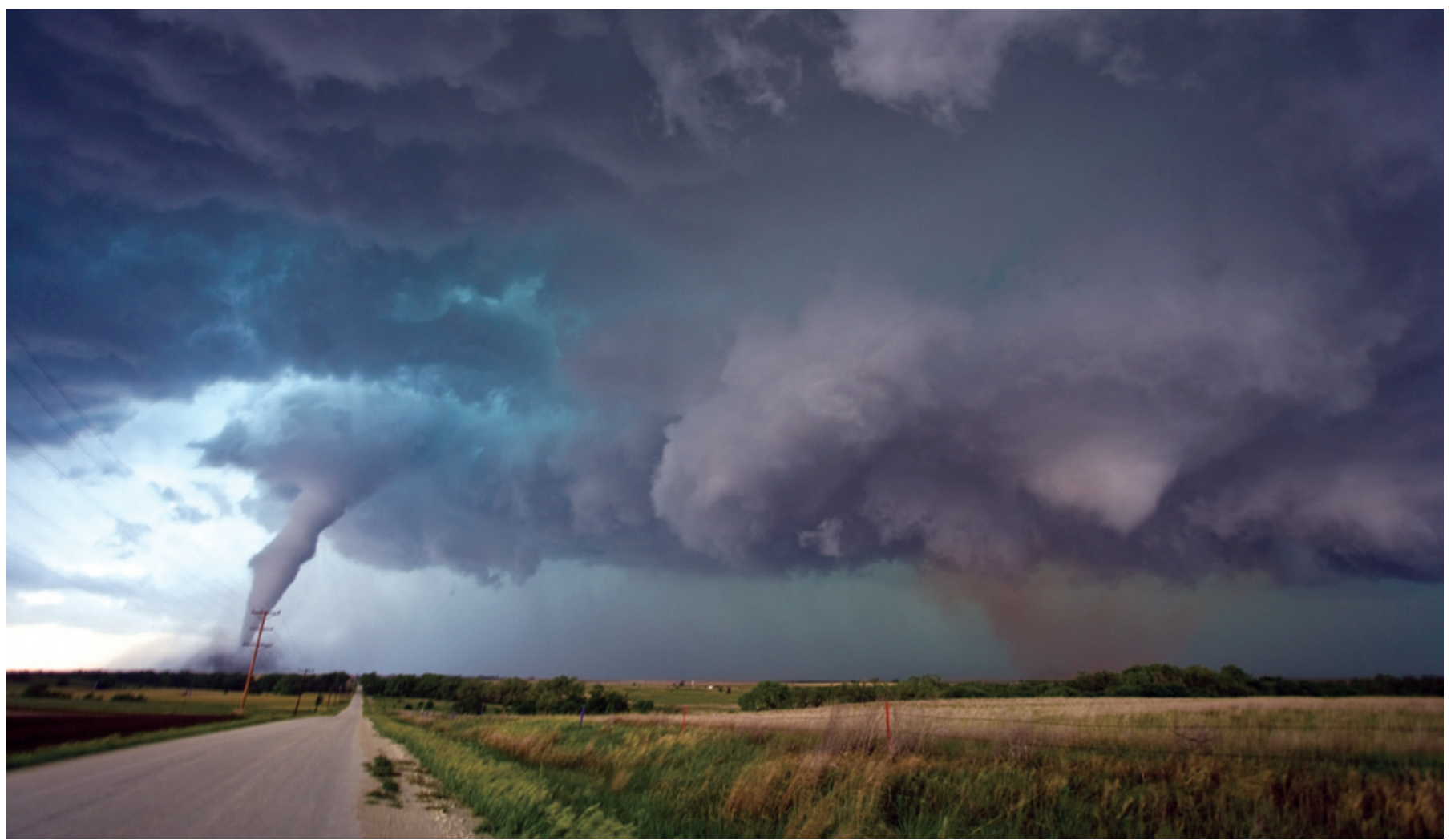

Two tornadoes near Zurich, Kansas. The central region of neighbouring Oklahoma is the most tornado-prone site in the world.

METEOROLOGY

\title{
On the twister trail
}

\section{Chuck Doswell enjoys a history of the passionate pioneers behind tornado science.}

$\mathrm{T}$

loday, most 'tornado chasers' are hobbyists who literally chase, observe and photograph tornadoes in the central plains of the United States, popularly known as Tornado Alley. Lee Sandlin's chronicle Storm Kings features the country's first tornado hunters - its pioneering meteorologists. Although they lacked the technology for the physical chase, they shared a passion for understanding tornadoes, a feeling I share.

We now know that most tornadoes form from rotating storms called supercells, and result from a concentration of that rotation - in essence, what happens when a vortex forms in the water draining from a bathtub. The challenge for scientists today is to understand why so few storms produce tornadoes, and whether climate change will influence the frequency and intensity of these events.

From the early eighteenth to the early twentieth centuries, however - the period that dominates Storm Kings - the science of storms was in its infancy. Sandlin opens with US scientist and statesman Benjamin Franklin. Although best known for his investigation into the electrical nature of lightning,
Franklin was also interested in tornadoes. He witnessed one in Maryland in 1754, recognizing the rotation of its whirling winds.

Sandlin then jumps to the mid-nineteenth century and the vitriolic clash between James Espy, who developed a 'thermal inflow' theory of cyclones - in which warm air rises and

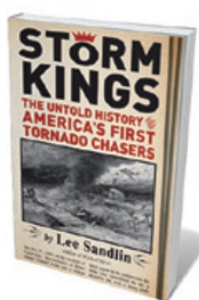

Storm Kings: The Untold History of America's First Tornado Chasers LEE SANDLIN Pantheon: 2013 304 pp. $\$ 26.95$ is replaced by air flowing inward - and William Redfield, who believed that cyclones comprised rotating winds. This 'weather war' raged until Redfield's death in 1857. It is now clear that both were correct up to a point, a helpful reminder that in scientific controversy opponents may be right about some things and wrong about others.

I particularly enjoyed Sandlin's review of the pioneering efforts of John Park Finley
(1854-1943), the first meteorologist to study tornadoes in any detail. Under the aegis of the US Army, Finley created the first tornado-spotter networks; wrote Tornadoes (1887), the first book on the subject; promoted the digging of underground tornado shelters; and issued the first experimental tornado forecasts and developed the first verification system to evaluate them. Finley's superiors eventually stopped his studies, which interfered with his military obligations. Subsequently, the word 'tornado' was banned in US public weather forecasts, as it was thought to cause public panic. The ban was lifted only in the early 1950s.

The quantitative risk of being hit by a violent tornado, even in central Oklahoma - the location with the highest frequency of them in the world - is so low that for any particular location, 1,000 years could pass without one. Yet someone, somewhere in the United States is hit every year. Finley understood the advisability of preparedness, and his view was validated in 2011 by the deaths of 553 people - the largest annual toll since 1925, when the Tri-State tornado devastated Missouri, 
Illinois and Indiana, as Sandlin describes.

Storm Kings touches briefly on Robert Miller and Ernest Fawbush, US Air Force officers who issued the first post-Finley tornado forecast, for Tinker Air Force Base in central Oklahoma on 25 March 1948. There is also an introduction to the groundbreaking tornado research of Theodore Fujita, and an overview of the era of modern storm chasing.

The United States, with the world's highest tornado frequency, is a mecca for such hobbyists. But tornadoes have been observed on every continent except Antarctica. The vulnerability of populations around the world to tornadoes is complex, involving factors such as population density, construction standards and storm severity, as well as frequency. In Bangladesh, for instance, single tornadoes have had very high death tolls: one, which hit Daulatpur and Saturia on 26 April 1989 , killed an estimated 1,300 people.

Sandlin's "storm kings" had an altogether simpler view of the risks, and were unaware of how tornadoes form in the outer rainbands of tropical cyclones or hurricanes. The potential for tornadoes to form as hurricanes make landfall is a real threat, but they tend to cause less damage than storm surges, which can wipe out miles of low-lying coastal areas.

We have also moved on in terms of our understanding of the psychological and behavioural aspects of preparedness: people have to believe that tornadoes might affect them personally. Preparation is not necessarily costly (an action plan and a stocked disaster kit are the basics), but attitude is key. Much remains to be learned about storms, not just about meteorology.

Storm Kings does contain technical errors and misconceptions. To take just one example, in discussing Espy's odd ideas about how water-vapour condensation powers thunderstorms, Sandlin mentions how temperature and pressure fall off with altitude. He refers to the calculation as "the saturated adiabatic lapse rate", but this term refers to the changes that occur under certain idealized assumptions, rather than real-world changes.

I have been a meteorologist interested in tornadoes for my entire career. Minor quibbles aside, I found Storm Kings a compelling history.

Chuck Doswell is Senior Research Scientist with the Cooperative Institute for Mesoscale Meteorological Studies affiliated with the University of Oklahoma. As part of his work as a consulting meteorologist, he has developed training materials for the National Weather Service and storm spotters. e-mail:cdoswell@earthlink.net

\section{Feel the photons}

\section{Joanne Baker revels in a show that celebrates the sensory power of light.}

I am in a pitch-black corridor, my senses straining. Feeling the wall as a guide, I take hesitant steps until I become aware of a dim glow. A shimmering wall of red bordered by a strip of pale turquoise beckons. Three radiant planes, set back at angles to one another and washed in shades of tomato and aquamarine, smack of abstract artist Mark Rothko's translucent layers. This is a painting in pure light.

James Turrell's engrossing Wedgework $V$ (1974), is a highlight of Light Show, an exhibition exploring the qualities of light at London's Hayward Gallery. From mathematical shapes traced in fluorescent tubes to an unlit incandescent bulb whose mirrorreflected filament is - puzzlingly - most definitely on, works by 22 artists grapple with light's ability to dazzle and deceive.

Perfectly paced strobe lighting freezes the motions of 27 tabletop fountains in DanishIcelandic artist Olafur Eliasson's Model for a Timeless Garden (2011). The arcing and burbling jets make a landscape of mercuric sculptures, organic forms that mesmerize as they slowly alter.

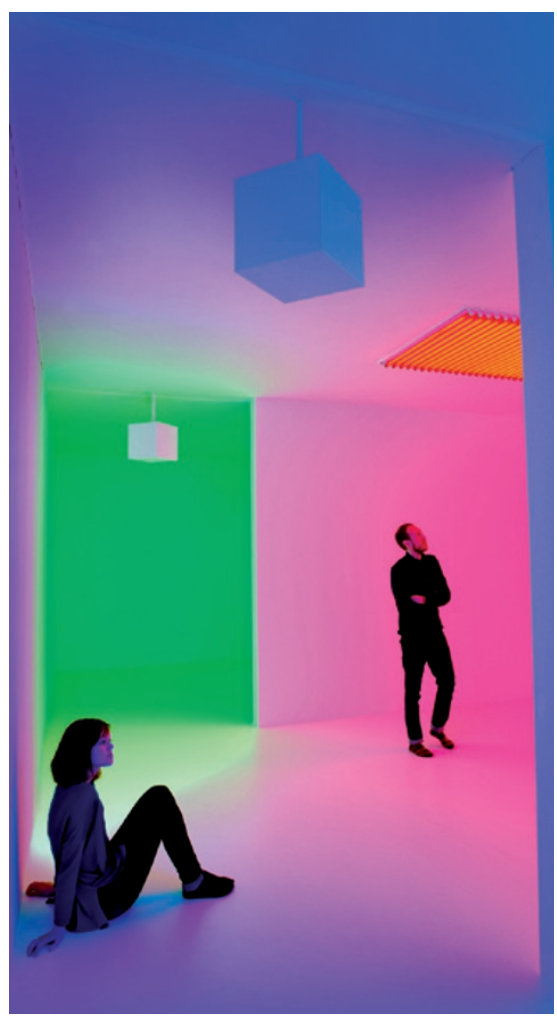

Chromosaturation by Carlos Cruz-Diez.
Shadows inspire Light Show Conrad Shawcross's HAYWARD GALLERY sculpture Slow Arc Southbank Centre, Inside a Cube IV London. (2009). A shifting latUntil 28 April 2013 tice of darkness cast on the gallery walls by a lamp moving within a wire cage is hypnotic, setting you off balance. Shawcross's work is influenced by chemist Dorothy Hodgkin, who described deducing protein structures using X-ray crystallography as akin to decoding the shape of a tree from the shadows of its leaves.

Plenty of the artists recreate natural illumination: a Las Vegas sunset captured in light boxes refashioned from shop signs; the spectrum of moonlight emitted by a bespoke halogen bulb; the phosphorescent flicker of cascades of light-emitting diodes (LEDs), which conjure up meteor showers or fireflies. Nancy Holt's row of circular perforations in a wall illuminated alternately from one side or the other recalls projections of the Sun's face or the transit of Venus across it.

Many installations are immersive. Visitors must don plastic shoe covers to step into a series of spaces bathed solely in blue, red or green. The monochromatic experience is disorienting, explains Venezuelan artist Carlos Cruz-Diez, because our retinas are used to perceiving a range of colours. Cone cells in the eye pick out each primary hue and other cells highlight opposing shades. Spend much time in a red room, and you start to appreciate even a tiny fleck of blue and green.

Theatrical lighting is used to great effect in Anthony McCall's You and I, Horizontal (2005). In a darkened room, puffs of mist bring the appearance of solidity to curved sheets of intense white light. Emanating from a point like an old film projector, the narrow sheets carve out a horizontal cone. Moving through this 'solid light' sculpture feels strangely subversive, like stepping through a wall.

Light Show does not expose much of the physical or optical qualities of light, as artists such as Eliasson have done elsewhere in works using prisms and optics. Revel instead in a playful and interactive look at the sensory side of light that illuminates as it entertains.

Joanne Baker is Senior Comment Editor at Nature in London. e-mail:j.baker@nature.com 